\title{
On the Process of Translation
}

These translations are the product of a collaboration between an American writer who has formally studied other Indian languages but not Telugu and a Telugu writer and scholar for whom some of these poems were recited in childhood, chanted by his father, a learned brahmin in a remote village where the way of life, in many ways, differed little from that of the period in which the poems were composed.

Our method brought us close to the oral tradition-of direct recitation and response—which still, despite the advent of print, exists very strongly as a feature of classical and popular Indian culture. V. Narayana Rao would write out these poems in Telugu, chant them in the traditional style, and supply the literal English meanings. Hank Heifetz would then compose an English poetic version. We would discuss this result, with Narayana Rao drawing on his memory-the true library of the classical Indian scholar-for illuminations and precisions. 
Explications of specific words would often open out into whole areas of the Telugu past. Phrases would be changed or emphases altered, as we entered more deeply into the meaning of the poems until finallyfor the best of them at least- the translation would reach a form, exhilarating us both, that seemed adequately to represent the original. Small changes might come up later, in private, but much of the work was done "publicly," by word of mouth, linking the process, for us, of contemporary translation to the archaic and fundamental learning mode of face-toface human dialogue. 